\title{
Wearable Devices and Al Techniques Integration to Promote Physical Activity
}

\author{
Dario Baretta \\ Department of Psychology \\ University of Milano-Bicocca \\ Milan - ITALY \\ d.baretta@campus.unimib.it \\ Fabio Sartori \\ Dept. of Informatics, Systems \\ and Communication \\ University of Milano-Bicocca \\ Milan - ITALY \\ sartori@disco.unimib.it

\section{Andrea Greco} \\ Department of Psychology \\ University of Milano-Bicocca \\ Milan - ITALY \\ andrea.greco@unimib.it

Riccardo Melen
Fabio Stella
Dept. of Informatics, Systems
and Communication
University of Milano-Bicocca
Milan - ITALY
melen@disco.unimib.it
stella@disco.unimib.it
Letizia Bollini
Marco D'addario
Patrizia Steca
Department of Psychology
University of Milano-Bicocca
Milan - ITALY
letizia.bollini@unimib.it
marco.daddario@unimib.it
patrizia.steca@unimib.it \\ Permission to make digital or hard copies of part or all of this work for \\ personal or classroom use is granted without fee provided that copies are \\ not made or distributed for profit or commercial advantage and that \\ copies bear this notice and the full citation on the first page. Copyrights \\ for third-party components of this work must be honored. For all other \\ uses, contact the Owner/Author. \\ Copyright is held by the owner/author(s). \\ MobileHCI '16 Adjunct, September 06-09, 2016, Florence, Italy \\ ACM 978-1-4503-4413-5/16/09. \\ http://dx.doi.org/10.1145/2957265.2965011
}

\begin{abstract}
Physical activity (PA) is considered one of the most important factors for the prevention and management of non-communicable diseases (NCDs). Mobile technologies offer several opportunities for supporting PA, especially if combined with psychological aspects, model-based reasoning systems and personalized human computer interaction. This still on-going research aims at developing a scalable framework that targets PA promotion among both clinical and nonclinical population, exploiting Bayesian Networks and Expert Systems to characterize and predict qualitative variables like self-efficacy. The expected outcomes are the collection and management of real-time behavioral and psychological data to define a personalized strategy for increasing PA.
\end{abstract}

\section{Author Keywords}

Bayesian Networks; Expert Systems; Wearable

Devices; Self-Efficacy; Behavior Change Techniques

\section{ACM Classification Keywords}

I.2.5 Expert Systems Tools and Techniques 


\section{Introduction}

Strong evidence shows that physical inactivity increases the risk of many adverse health conditions, including the world's major non-communicable diseases (NCDs) of cardiovascular diseases, type 2 diabetes, and breast and colon cancers, and shortens life expectancy. Elimination of physical inactivity would remove between $6 \%$ and $10 \%$ of the major NCDs. Since much of the world's population is inactive, this presents a major public health problem [1]. Mobile technologies offer several opportunities for supporting PA. We can monitor people continuously and give feedback messages at any moment in time, taking the specific context into account. However, existing apps don't usually consider either user's psychological aspects or their relationship with user's behaviour. Moreover, it remains unclear if existing apps are successful in achieving long-term behaviour change. Also, when taking a more technical perspective, it's clear that Apps promoting physical activity don't make use of the full potential of wearables' technology. We identified 3 main points of view that may contribute to make technology, and in particular mobile Apps, a more powerful tool for the PA increasing:

1. Given that people carry smartphones and can access data anywhere and anytime, physical activity behaviour change promotion apps offer the opportunity to provide tailored feedback and advice at the appropriate time and place. Therefore, apps offer new opportunities to deliver individually tailored interventions, including real-time assessment and feedback that are more likely to be effective.

2. Apps promoting PA are not often grounded in a specific psychological theory of behaviour change and their effectiveness is not evidence-based [2]. However, previous research suggests that the use of specific Behaviour Change Techniques (BCTs) [3] to address behavioural determinants conceptualized in behaviour change theory, is linked to effectiveness in PA promotion and in PA self-efficacy increasing [4-5]. As far as concerning self-efficacy, many references [6-7] suggest that interventions that aim to increase PA self-efficacy are related to the selection of challenging goals and to an increasing in PA behaviour.

3. Once that the psychological review highlighted the BCTs that influence the increasing of PA, App developers have to translate such psychological requirements into App functionalities and features. In this process, it's fundamental to consider how to make technology persuasive tool that influence patient attitudes and behaviours. The Apps should propose functionalities and features that are easyto-use, user-friendly, user-oriented, unobtrusive, entertaining and relevant [8].

Recent researches [9] highlighted the importance to simultaneously consider these points of view in order to implement more effective and tailored intervention programs. The main contribution of this paper is to introduce an innovative conceptual and computational framework that combines intelligent data interpretation and elaboration with behavioural and psychological variables. The proposed approach is based on the Higher-Level Knowledge Artifact (HLKA) model [10], through which the both behavioural (e.g. the heart rate) and psychological (e.g. the self-efficacy level) variables are integrated to suggest the amount and distribution of PA overt the time. The HLKA exploits ontologies, Bayesian networks and rule-based systems 
to support in a complete way the knowledge acquisition, representation and use process.

\section{Method}

In our preliminary study, we focused on the association between self-efficacy and goal-setting in order to define a goal-setting strategy based on PA self-efficacy and past PA behavior. The psychological model focuses on levels of self-efficacy that change dynamically in response to day-to-day achievements of personalized goals. The increasing in self-efficacy is also supported by the implementation of specific BCTs (e.g. feedback, monitoring, planning, problem solving). Given a person, possibly in the 35-45 years old range, who is not affected by any particular disease and characterized by very low level of physical activity, we want to suggest a personalized plan to increase such level in order to obtain benefits in the future, like reducing the risks of cardio-vascular diseases.

Figure 1 shows a sketch of the conceptual model of the framework: the evaluation of individuals' PA depends on the metabolic equivalent (MET) calculus. METs can be valued according to the following formula, as proposed in [11]: $M E T=4 *$ Time $^{\text {MPA }}+8^{*}$ Time $^{I P A}$, where Time $^{\mathrm{MPA}}$ andTime ${ }^{\mathrm{IPA}}$ represent the timing of Moderate and Intense Physical Activity, respectively. The characterization of a PA as intense or moderate depends on the person heart rate registered during the PA execution, as shown in Figure 2, where the computational model of the framework is presented.

The computational model has been implemented as an Android app using the Google Fit platform to monitor PA (in particular, the user heart rate). Given a set of available wearables (like smartwatches, smartphones, bracelets), data collected through them

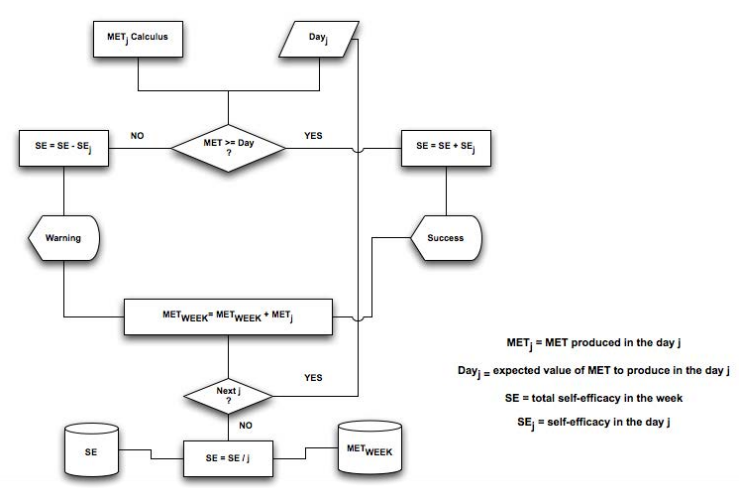

Figure 1: The psychological model for evaluating PA level

are elaborated by the AI module of the system, composed of a Bayesian network manager (BNM) and an Expert System manager (ESM). The BN implements the rules extraction for the ES according to the HLKA model [10]. The BNM allows estimating psychological variables like self-efficacy, whose value is usually determined by the expert on the basis of subjective knowledge acquisition campaigns; the ESM allows implementing the expert reasoning process using both quantitative and qualitative data.

\section{Expected results and discussion}

Thanks to the over mentioned system we expect that the processing of input data (PA self-efficacy and past PA behavior) may produce personalized and suitable output data (PA goal-setting). In this direction, it's possible to predict the likelihood that any specific goal can be achieved by a particular user, and to predict the impact of success (or failure) in achieving a goal on 


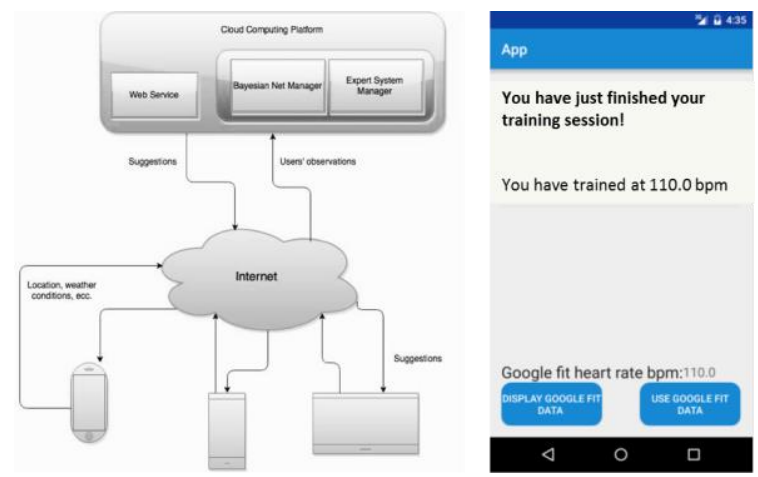

Figure 2: On the left, the framework cloud-based architecture; on the right, the GUI of the prototype to collect real-time data.

future levels of self-efficacy. Another expected result is the development of a huge database of objective data about the PA accomplishment, in order to improve the analysis of user feedbacks with less subjective information. These data could be useful to address future directions of our research, possibly extending the applicability of our approach to the medical domain.

\section{References}

1. I-Min Lee, Eric J. Shiroma, Felipe Lobelo, Pekka Puska, Steven N. Blair, and Peter T. Katzmarzyk, Lancet Physical Activity Series Working Group. 2012. Effect of physical inactivity on major non communicable diseases worldwide: an analysis of burden of disease and life expectancy. Lancet Lond. Engl. 380, 219-229.

2. Sherry Pagoto and Gary G. Bennett. 2013. How behavioral science can advance digital health. Transl. Behav Med;3(3):271-6.

3. Susan Michie, Michelle Richardson, Marie Johnston, Charles Abraham, Jill Francis, Wendy Hardeman, Martin P. Eccles, James Cane, and Caroline E.
Wood. 2013. The behavior change technique taxonomy (v1) of 93 hierarchically clustered techniques: Building an international consensus for the reporting of behavior change interventions. Ann Behav Med. Aug;46(1):81-95.

4. Susan Michie, Charles Abraham, Craig Whittington, John McAteer, and Sunjai Gupta. 2009. Effective techniques in healthy eating and physical activity interventions: A meta-regression. Health Psychol. Nov;28(6):690-701.

5. Ellinor K. Olander, Helen Fletcher, Stefanie Williams, Lou Atkinson, Andrew Turner, and David P. French. 2013. What are the most effective techniques in changing obese individuals' physical activity self-efficacy and behaviour: A systematic reviewand meta-analysis. Int J Behav Nutr Phys Act.;10:29.

6. Albert Bandura. 1997. Self-efficacy: The exercise of control. New York: Freeman

7. Stefanie Williams and David P. French. 2011. What are the most effective intervention techniques for changing physical activity self-efficacy and physical activity behaviour-and are they the same? Health Educ Res. Apr;26(2):308-322.

8. Julia E. W. C. Van Gemert-Pijnen, Oscar Peters, and Hans C. Ossebaard. 2013. Improving eHealth. The Hague: Boom.

9. Michel C. A. Klein, Adnan Manzoor, Anouk Middelweerd, Julia S. Mollee, and Saskia J. teVelde. 2015. Encouraging Physical Activity via a Personalized Mobile System. In IEEE Internet Computing, 19(4), 20-27.

10. Riccardo Melen, Fabio Sartori, and Luca Grazioli.2015. Modeling and understanding timeevolving scenarios. JSCI 13, 5: 62-67.

11. Thimothy Armstrong and Ruth Bonita. 2003. Capacity building for an integrated non communicable disease risk factor surveillance system in developing countries. Ethn Dis. 13:2-13. 